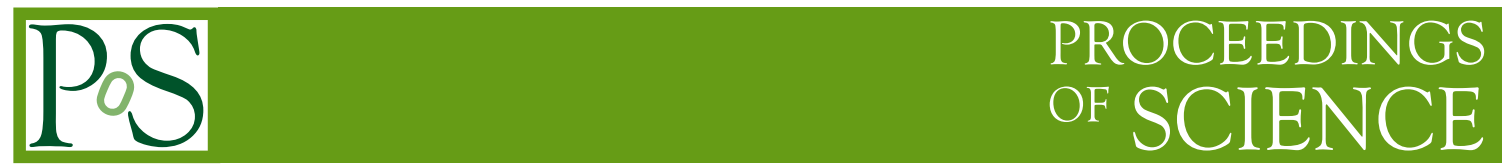

\title{
Quark number susceptibilities at intermediate coupling
}

\author{
Nan Su* \\ Fakultät für Physik, Universität Bielefeld, D-33615 Bielefeld, Germany \\ E-mail: nansu@physik.uni-bielefeld.de
}

We present recent results of the second and fourth order quark number susceptibilities from two schemes of resummed perturbation theory: 1) hard-thermal-loop perturbation theory at one-loop order; 2) resummation of the four-loop finite density equation of state derived using a dimensionally reduced effective theory. We then compare the results with recent high precision lattice data from BNL-Bielefeld and Wuppertal-Budapest collaborations.

Xth Quark Confinement and the Hadron Spectrum,

October 8-12, 2012

TUM Campus Garching, Munich, Germany

${ }^{*}$ Speaker. 


\section{Introduction}

The responses of the QCD pressure to the change of quark chemical potentials are described by quark number susceptibilities defined by

$$
\left.\chi_{i j k \ldots}(T) \equiv \frac{\partial^{n} p\left(T,\left\{\mu_{f}\right\}\right)}{\partial \mu_{i} \partial \mu_{j} \partial \mu_{k} \cdots}\right|_{\mu_{f}=0},
$$

where the indices $i, j, k, \ldots$ refer to different quark flavors. These quantities are of great importance due to the fact that they are closely related to fluctuations of conserved charges which signal the QCD phase transition. More interestingly, these quantities are free of the notorious sign problem, and therefore can be accessed on the lattice (see e.g. [1,2] and references therein for recent studies). Besides, extensive analytic work on susceptibilities has been carried out within unresummed perturbation theory [3, 4], the hard-thermal-loop (HTL) approximation [5, 6, 7, 8, 9], the analytically tractable large- $N_{f}$ limit of QCD [10,11], and even the gauge/gravity duality [12].

The strength of the QCD running coupling in the RHIC and LHC energy regimes is on the order of $g \sim 2$, which is some intermediate value neither infinitesimally small nor infinitely large. It is clear that a quantitative description of the quark-gluon plasma in this intermediate coupling regime necessitates the use of nonperturbative techniques, while it is also of theoretical interest to explore to what extent resummed perturbation theory would be able to able to provide a good approximation. In this proceedings, we focus on the second and fourth order quark number susceptibilities obtained from state-of-the-art resummed perturbative calculations. To this end, we address results from two separate resummation schemes: 1) Hard-thermal-loop perturbation theory (HTLpt) at one-loop order [13] which amounts to shifting the perturbative expansion from being around an ideal gas of massless particles which is the physical picture of the weak-coupling expansion, to being around a gas of massive quasiparticles which are the more appropriate physical degrees of freedom at high temperature and/or density (see [14] for a review); 2) Application of the resummation scheme of $[15,16]$ to the four-loop $\mu \neq 0$ equation of state derived in [3] using a dimensionally reduced (DR) effective theory $[17,18]$ which leads to a significant improvement in the convergence and renormalization scale dependence of the results. In the next section, we will present our results and compare them with recent high precision lattice data in the intermediate coupling regime, while we refer to [19] for the formalisms and calculation details.

\section{Results and discussions}

In this section, we will concentrate on the phenomenologically relevant case of three dynamical quark flavors. All the results in [19] are obtained using dimensional regularization in the $\overline{\mathrm{MS}}$ scheme, denoting the renormalization scale by $\bar{\Lambda}$. The central value of $\bar{\Lambda}$ is determined by applying the Fastest Apparent Convergence (FAC) criterion to the three-dimensional gauge coupling $g_{3}$, resulting in $\bar{\Lambda}_{\text {central }} \approx 1.445 \times 2 \pi T$ [18], around which a factor of 2 is varied in order to test the sensitivity of the result with respect to this choice. For the QCD running coupling, we use one-loop perturbative expression in the HTLpt result and two-loop one in the DR case, in accordance with the conventional criterion that the uncertainties originating from the running coupling should not exceed those due to the perturbative computation itself. For the choice of the QCD scale 


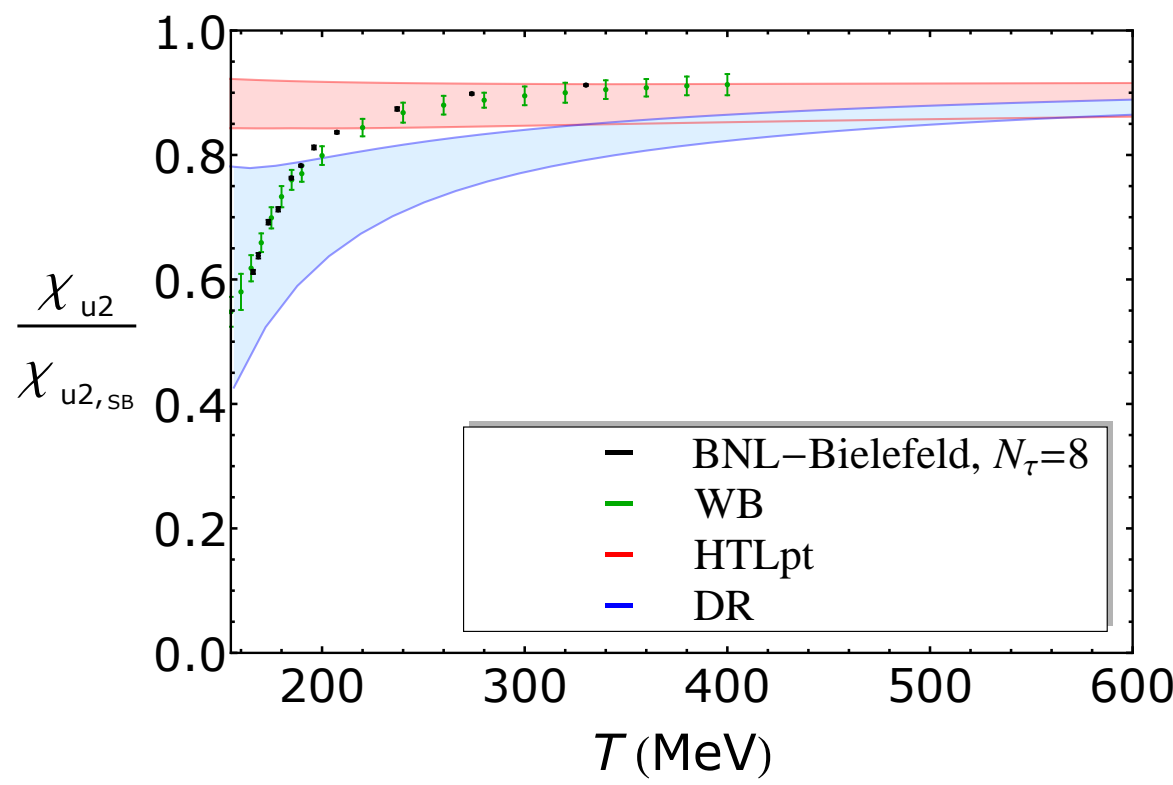

Figure 1: A comparison of our HTLpt (red) and DR (blue) results of the second order quark number susceptibility $\chi_{\mathrm{u} 2}$ with the latest lattice results of the BNL-Bielefeld [21, 22] (black) and Wuppertal-Budapest (WB) [2] (green) collaborations.

$\Lambda_{\overline{\mathrm{MS}}}$, we use a recent lattice determination of the running coupling at a reference scale of $1.5 \mathrm{GeV}$ [20]. Requiring that our one- and two-loop running couplings agree with this, we obtain the values $176 \mathrm{MeV}$ and $283 \mathrm{MeV}$ in these two cases, respectively. To access the uncertainties of these numbers, we vary the value of $\Lambda_{\overline{\mathrm{MS}}}$ around these numbers by $30 \mathrm{MeV}$, which is somewhat larger than the reported lattice error bars.

In Figure 1, we show our results of the second order quark number susceptibility $\chi_{\mathrm{u} 2} \equiv \chi_{u u}$, normalized by its noninteracting Stefan-Boltzmann (SB) limit value $\chi_{\mathrm{u} 2, \mathrm{SB}}=T^{2}$. The results are compared with the latest $N_{\tau}=8$ lattice data of the BNL-Bielefeld collaboration (black) using the HISQ action [21, 22], and the recent continuum extrapolated lattice data from the WuppertalBudapest (WB) collaboration (green) [2]. The bands of the HTLpt (red) and DR (blue) results are obtained by varying both $\bar{\Lambda}$ and $\Lambda_{\overline{\mathrm{MS}}}$ as described in the last paragraph. The widths of the bands demonstrate that our results have a rather mild dependence on the chosen renormalization scale as well as the QCD scale. For instance, a comparison of the DR band with the unresummed four-loop result of [3] shows a reduction of the uncertainty by a factor close to 10 in this temperature range. Our two results are in addition in reasonably good agreement with each other at high temperatures, deviating in a significant way only below $400 \mathrm{MeV}$. In this low temperature regime, we see that the HTLpt result overlaps with the lattice data down to $T \sim 250 \mathrm{MeV}$, while the DR one - despite qualitatively reproducing the trend of the lattice results - consistently underestimates them by approximately $10 \%$ (until even lower temperatures, where the agreement is most likely accidental).

In Figure 2, we show our results of the fourth order quark number susceptibility $\chi_{\mathrm{u} 4} \equiv \chi_{\text {ииии }}$, again scaled by its SB limit $\chi_{\mathrm{u} 4, \mathrm{SB}}=6 / \pi^{2}$. The continuum extrapolated WB lattice data here 


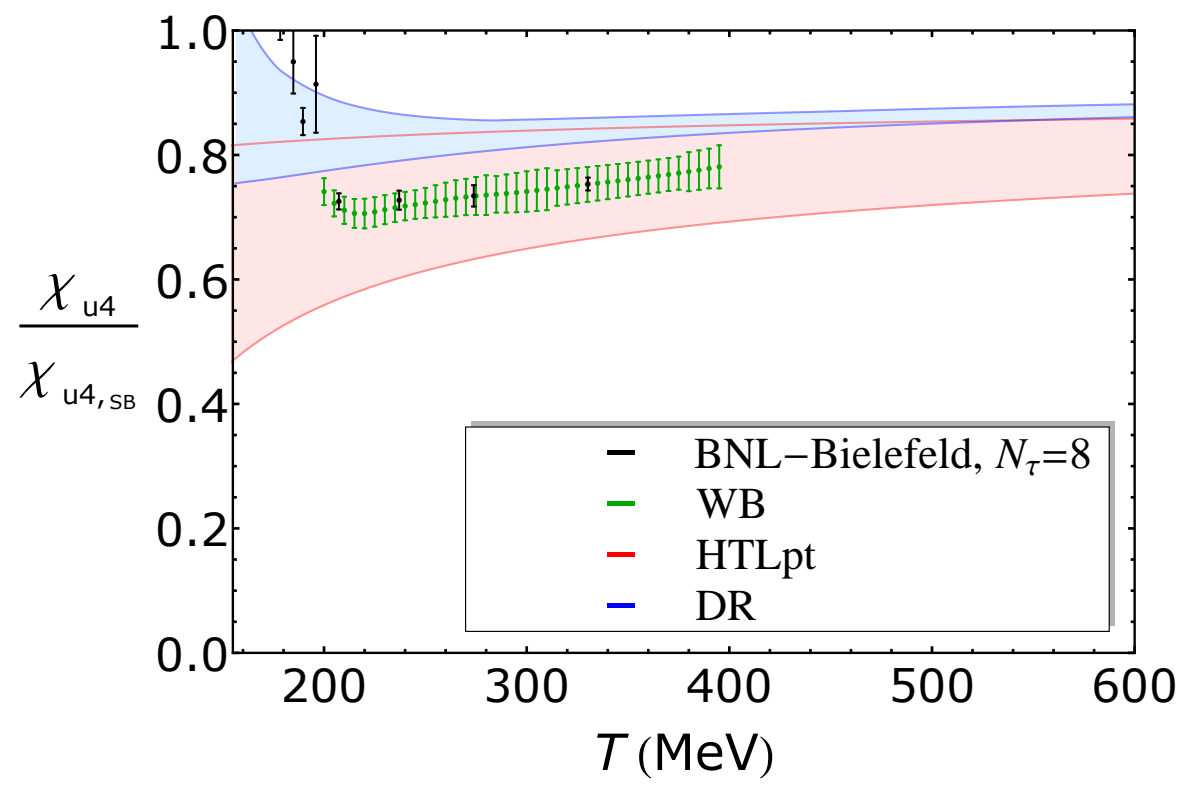

Figure 2: A comparison of our HTLpt (red) and DR (blue) results of the fourth order quark number susceptibility $\chi_{\mathrm{u} 2}$ with the latest lattice results of the BNL-Bielefeld [21, 22] (black) and Wuppertal-Budapest (WB) [23] (green) collaborations.

is their latest from [23], while the BNL-Bielefeld lattice data are again obtained using the HISQ action with $N_{\tau}=8$ [21,22]. It is clearly seen that both data sets are inside the HTLpt band until $T \sim 200 \mathrm{MeV}$. The DR prediction is again seen to reproduce the qualitative trend of the lattice data, but there is once more a roughly $10 \%$ offset between the two predictions at the most relevant temperatures. Curiously, this time the DR band lie above the lattice data, which in particular implies that there is no choice of parameters such as $\bar{\Lambda}$ and $\Lambda_{\overline{\mathrm{MS}}}$, for which both the second and fourth order susceptibilities would match the lattice predictions.

A particularly interesting aspect of our results can be found in the temperature range of 250$500 \mathrm{MeV}$, where both of our perturbative predictions as well as the lattice data indicate that the (appropriately normalized) second and fourth order susceptibilities are relatively slowly varying functions of $T$. However, whereas the DR results predict that both quantities are roughly $20 \%$ below their SB limit at these values of $T$, in the lattice and HTLpt results there is a clear difference between $\chi_{\mathrm{u} 2}$ and $\chi_{\mathrm{u} 4}$, suggesting that the former reaches the SB limit much faster than the latter. This difference is particularly prominent in Figure 3, where we plot the ratio of the two susceptibilities, $T^{2} \chi_{\mathrm{u} 4} / \chi_{\mathrm{u} 2}$, for which the $T \rightarrow \infty$ limit is $6 / \pi^{2}$ (dashed line). From here, we see a clear discrepancy between the highly robust DR prediction, resulting from an almost perfect cancelation of the renormalization and $\Lambda_{\overline{\mathrm{MS}}}$ dependence in the ratio, and the lattice results. At the same time, the HTLpt band, still exhibiting mild scale dependence, is seen to be consistent with the lattice data over a remarkably large temperature range. It should be extremely interesting to attempt to explain this behavior, both by studying the potential numerical effect that including the unknown $\mathscr{O}\left(g^{6}\right)$ term may have on the DR result, and by deriving a two-loop HTLpt prediction for the susceptibilities from continuing the recent work of [24]. 


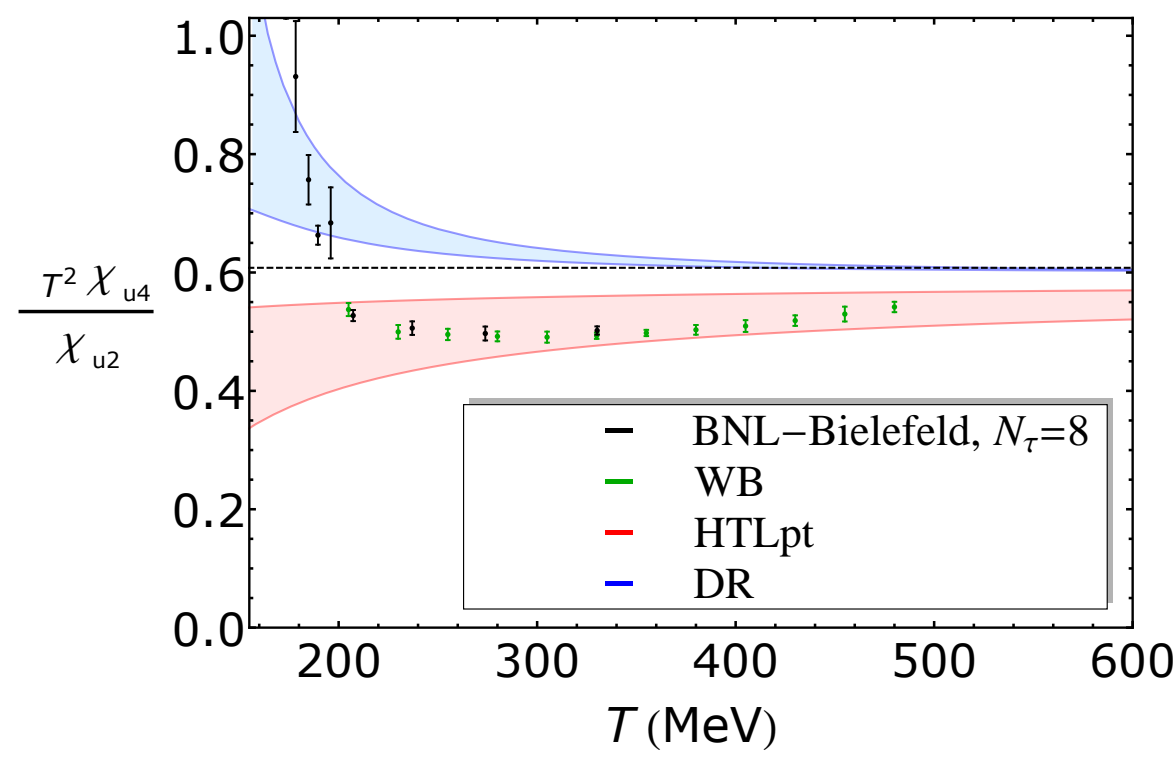

Figure 3: The ratio of the results shown in the previous two figures. This time, the quantity is not normalized by the $\mathrm{SB}$ value, but rather approached the value $6 / \pi^{2}$ (dashed line) at high temperatures.

Unlike lattice calculations, perturbation theory works optimally at very high temperatures and thus offers a way to connect the results obtained around the phase transition to arbitrarily high energies. More importantly, in contrast to lattice, perturbative calculations are free from the sign problem, ad therefore can be straightforwardly generalized to finite density. Hopefully, our results will find phenomenological applications in the study of the current and future heavy ion data from RHIC, LHC, and FAIR.

\section{Acknowledgments}

The work was done in collaboration with Jens O. Andersen, Sylvain Mogliacci, and Aleksi Vuorinen. The author was supported by the Postdoctoral Research Fellowship of the Alexander von Humboldt Foundation.

\section{References}

[1] A. Bazavov et al. (HotQCD Collaboration), Fluctuations and correlations of net baryon number, electric charge, and strangeness: A comparison of lattice QCD results with the hadron resonance gas model, Phys. Rev. D 86, 034509 (2012). [arXiv:1203.0784 [hep-lat] ].

[2] S. Borsanyi et al., Fluctuations of conserved charges at finite temperature from lattice QCD, J. High Energy Phys. 01 (2012) 138 [arXiv:1112.4416 [hep-lat]].

[3] A. Vuorinen, Quark number susceptibilities of hot QCD up to $g^{6} \ln g$, Phys. Rev. D 67, 074032 (2003) [hep-ph/0212283]. 
[4] A. Vuorinen, Pressure of QCD at finite temperatures and chemical potentials, Phys. Rev. D 68 , 054017 (2003) [hep-ph/ 0305183$].$

[5] J. P. Blaizot, E. Iancu, and A. Rebhan, Quark number susceptibilities from HTL resummed thermodynamics, Phys. Lett. B 523, 143 (2001) [hep-ph / 0110369 ].

[6] J. P. Blaizot, E. Iancu, and A. Rebhan, Comparing different hard-thermal-loop approaches to quark number susceptibilities, Eur. Phys. J. C 27, 433 (2003) [hep-ph/ 0206280 ].

[7] P. Chakraborty, M. G. Mustafa, and M. H. Thoma, Quark number susceptibility in hard thermal loop approximation, Eur. Phys. J. C 23, 591 (2002) [hep-ph/0111022].

[8] P. Chakraborty, M. G. Mustafa, and M. H. Thoma, Quark number susceptibility, thermodynamic sum rule, and hard thermal loop approximation, Phys. Rev. D 68, 085012 (2003) [hep-ph/0303009].

[9] N. Haque, M. G. Mustafa, and M. H. Thoma, Conserved density fluctuation and temporal correlation function in hard thermal loop perturbation theory, Phys. Rev. D 84, 054009 (2011) [arXiv:1103.3394 [hep-ph]].

[10] A. Ipp and A. Rebhan, Thermodynamics of large- $N_{f} Q C D$ at finite chemical potential, J. High Energy Phys. 06 (2003) 032 [hep-ph/0305030].

[11] A. Ipp, A. Rebhan, and A. Vuorinen, Perturbative QCD at nonzero chemical potential: Comparison with the large- $N_{f}$ limit and apparent convergence, Phys. Rev. D 69, 077901 (2004). [hep-ph/0311200].

[12] J. Casalderrey-Solana and D. Mateos, Off-diagonal flavour susceptibilities from AdS/CFT, J. High Energy Phys. 08 (2012) 165. [arXiv:1202.2533 [hep-ph]].

[13] J. O. Andersen, E. Braaten, and M. Strickland, Hard-Thermal-Loop Resummation of the Free Energy of a Hot Gluon Plasma, Phys. Rev. Lett. 83, 2139 (1999) [hep-ph / 9902327 ].

[14] N. Su, A Brief Overview of Hard-Thermal-Loop Perturbation Theory, Commun. Theor. Phys. 57, 409 (2012) [arXiv:1204.0260 [hep-ph]].

[15] J. P. Blaizot, E. Iancu, and A. Rebhan, On the apparent convergence of perturbative QCD at high temperature, Phys. Rev. D 68, 025011 (2003) [hep-ph/ 0303045$].$

[16] M. Laine and Y. Schröder, Quark mass thresholds in QCD thermodynamics, Phys. Rev. D 73, 085009 (2006). [hep-ph/0603048].

[17] E. Braaten and A. Nieto, Effective field theory approach to high-temperature thermodynamics, Phys. Rev. D 51, 6990 (1995) [hep-ph/9501375].

[18] K. Kajantie, M. Laine, K. Rummukainen, and M. E. Shaposhnikov, 3d $S U(N)+$ adjoint Higgs theory and finite temperature QCD, Nucl. Phys. B 503, 357 (1997) [hep-ph/ 9704416 ].

[19] J. O. Andersen, S. Mogliacci, N. Su, and A. Vuorinen, Quark number susceptibilities from resummed perturbation theory, arXiv:1210.0912 [hep-ph].

[20] A. Bazavov et al., Determination of $\alpha_{s}$ from the QCD static energy, Phys. Rev. D 86, 114031 (2012) [arXiv:1205.6155 [hep-ph]].

[21] C. Schmidt, Baryon number and charge fluctuations from lattice QCD, arXiv: 1212.4278 [hep-lat].

[22] C. Schmidt, QCD bulk thermodynamics and conserved charge fluctuations with HISQ fermions, arXiv:1212.4283 [hep-lat]. 
[23] S. Borsanyi, Thermodynamics of the QCD transition from lattice, arXiv: 1210.6901 [hep-lat]; The errors of the data are obtained from private communication.

[24] N. Haque, M. G. Mustafa, and M. Strickland, Two-loop HTL pressure at finite temperature and chemical potential, arXiv:1212.1797 [hep-ph]. 